\title{
Lifestyle change and type 2 diabetes mellitus risk factors among students of the medical faculty of Conakry (Guinea)
}

\author{
Vitaly T CHIRKOV ${ }^{*}$, Naby Moussa BALDE ${ }^{2}$ and Vladimir CHIRKOV \\ ${ }^{1}$ Sport and Social Sciences Laboratory (EA1342), University of Strasbourg, Strasbourg, France \\ ${ }^{2}$ Department of Endocrinology, University Hospital, Conakry, Guinea \\ ${ }^{3}$ Faculty of Medicine, University of Conakry, Conakry, Guinea
}

\begin{abstract}
Objective: The evolution of the type 2 diabetes mellitus (T2DM) is more than alarming in West Africa. Studies on the subject are rare in Guinea. The aim of this study was therefore to evaluate its main risk factors, including family history, Body Mass Index (BMI), malnutrition and physical activity.

Method: This study was carried out in June 2015 and concerned 970 students at the Medical Faculty of the University of Conakry (average age was 22.5 years, \pm 2.7 ). The survey and the height and weight measurements were made during the lab courses in attendance of the investigators.

Results: $22.2 \%$ of respondents reveal cases of diabetes in their families including $10.1 \%$ among their close relatives. Furthermore, $31.3 \%$ of them have one of the risk factors of the T2DM mentioned above, $44.6 \%$ have two risk factors, $17.5 \%$ and $2.3 \%$ have respectively three and four of them.

Conclusion: The risk factors related to the lifestyle, such as physical inactivity and malnutrition are the main factors associated to type 2 diabetes mellitus. While the modern lifestyle impacts the food transition, increasing the proportion of sugar and fat, the traditions provide no importance to physical exercise and promote the ideal of a fat body, especially for women.
\end{abstract}

\section{Introduction}

Considered as one of the major challenges to public health, the type 2 diabetes mellitus [T2DM] now expands on all continents [1]. By 2025, the most significant increase of its prevalence will be recorded in developing countries that will contain nearly $75 \%$ of patients with diabetes in the world [2]. In West Africa, its expansion represents a real epidemiological transition to noncommunicable diseases [3]. Therefore, faced with HIV/AIDS, malaria, tuberculosis and recently hemorrhagic fever Ebola, some countries in this geographical area are now facing new medical threats related to changes in lifestyle such as physical inactivity and malnutrition. In these countries, the dominant structure of health systems that is organized around the management of infectious diseases and emergencies, has reached its limits with the emergence of noncommunicable diseases including T2DM [4].

In Guinea, the data collected in the T2DM care units are incomplete and probably underestimate the extent of the phenomenon. A first survey on the prevalence of diabetes and its risk factors seems to confirm the extent of the problem and the disparities between urban and rural areas [5]. A random sample of 1537 subjects aged over 35 years reported an overall prevalence of $6.1 \%$ diabetes. Its frequency was twice as high in urban areas compared to rural areas. The second survey on noncommunicable diseases including T2DM occurred in 2009. It was conducted in Lower Guinea Conakry and following the WHO STEPS protocol [6]. The survey focused on 2500 subjects aged from 15 to 64 . The prevalence of diabetes varied across the populations from $3.5 \%$ to $6.8 \%[5]$.
In our present investigation, we studied the young Guinean population, including medical students of the University of Conakry. Indeed, in recent decades, T2DM tends to "rejuvenate" not only in Western countries but also in developing countries. This trend coincides with the increase in obesity and inactivity among young adults in particular [7]. The T2DM is a disease that gradually develops as a result of the competition of several risk factors, so it is important to identify them as soon as possible. Especially, it is the combination of several factors that contributes to the development of T2DM. Some of these factors are unchangeable as sex, age, genetics or ethnicity. However, the involvement of sex as a risk factor remains uncertain: no significant difference in the Gulf region [8], in West Africa [9] and in Guinea [5], but more pronounced among men in France [10] and Canada [11]. On the other side, there can be no doubt on the influence of genetic factors and family history [12]. Thus, almost $30 \%$ of type 2 diabetes have at least one diabetic parent. This fact indicates a genetic predisposition, but the fact remains that the adoption of similar lifestyle could be a factor associated to the link with family history [13]

Ethnicity may also play a significant role. Epidemiological studies

Correspondence to: Vitaly TCHIRKOV, Sport and Social Sciences Laboratory (EA1342), University of Strasbourg, Strasbourg, France, E-mail: vitalytch@hotmail.com

Key words: Type 2 diabetes mellitus, Guinea, BMI, malnutrition, physical activity

Received: January 03, 2017; Accepted: January 31, 2017; Published: February 04, 2017 
suggest the existence of ethnic differences in insulin sensitivity [14]. However, studies in West Africa are scarce and do not allow the estimation of the level of insulin sensitivity of different local populations. Among the risk factors depending on the behavior and the lifestyle, we can mainly name the overweight and physical inactivity, which induce respectively $5 \%$ and $6 \%$ of mortality over the World [15]. It is well established that physical inactivity is one of the major risk factors and the main cause of $27 \%$ of diabetes cases [16]. Furthermore, according to WHO, the emergence of overweight and obesity in developing countries is the direct cause of the explosion of T2DM. This phenomenon is closely linked to the nutritional transition of traditional diets towards unbalanced food [17]. Physical activity in turn reduces the risk of development of T2DM and slowed disease progression by increasing insulin sensitivity [18].

Based on the above, we believe that the lifestyle of young adults living in the capital is evolving, so that this category may develop T2DM over time. The main risk factors are the lack of physical activity characterized by a low level of sports and walking perimeter; changing eating habits that lead to a higher frequency of high glycemic index foods; weight gain, partly reinforced by culture, local traditions and family diabetes history.

\section{Method}

The study was carried out in June 2015 and concerned 970 students currently enrolled between 1st and 6th year of study at the medical faculty of the Gamal Abdel Nasser University of Conakry. The average age was $22.5 \pm 2.7$ years and its minimum and maximum values were situated between 17 and 37 years (Table 1).

The questionnaire contained 22 open-ended and closed-ended questions grouped into several items such as general data, pathologies, diabetes family history, home and living environment, nourishment, physical activity and sports, and the subjective perception of bodyweight. The survey and the height and weight measurements were made during the lab courses in attendance of the investigators.

The Body Mass Index (BMI) was categorized into 5 standard intervals: extremely thin (less than $16.5 \mathrm{~kg} / \mathrm{m}^{2}$ ), thin (between 16.5 and $18.5 \mathrm{~kg} / \mathrm{m}^{2}$ ), normal index (between 18.5 and $25 \mathrm{~kg} / \mathrm{m}^{2}$ ), overweight (more than 25 and less than $30 \mathrm{~kg} / \mathrm{m}^{2}$ ) and obesity (over $30 \mathrm{~kg} / \mathrm{m}^{2}$ ). The daily walking perimeter was estimated individually using the detailed map of Conakry.

All statistical analyzes were carried out with the SPAD 5.5 software. The significance of $\mathrm{p} \leq 5 \%$ limit was respected. Thus, when the value test " $r$ " was greater than " 2 " in absolute terms, the difference was considered significant [19]. From an ethical standpoint, our study did not require review under Guinean National Ethical Comity for Health Research.

\section{Results}

The data collected using the questionnaire shows that $79.6 \%$ of surveyed students are Guinean and come from the capital Conakry (22\%) or from four geographical regions: Forest Guinea (18.7\%), Lower Guinea (14.5\%), Middle Guinea (13.1\%) and Upper Guinea (11.3\%). Slightly more than $20 \%$ of the students are foreign and come mainly from the neighboring countries such as Mali, Ivory Coast, Cameroon and Mauritania. The majority (65.7\%) lives with parents or relatives, $28.4 \%$ live alone and $5.9 \%$ live in couples. More than $99 \%$ live through family support, less than $1 \%$ has their own income. Among the parental professions, the employees represented $30.5 \%, 27.5 \%$ are executives and $27.2 \%$ are entrepreneurs (traders, artisans \& others). Thus $85 \%$ of respondents come from relatively wealthy families. Farmers and workers constitute respectively only $10.8 \%$ and $2.3 \%$, reflecting the elitist character of the medical higher education in Guinea.

The majority (79.9\%) of the surveyed students don't show any obvious diseases or disability. Among $20.1 \%$ of those who reported health problems, $51.1 \%$ suffer from gastrointestinal disorders, $21.2 \%$ from respiratory disorders, $17.1 \%$ from motor disorders, $8.3 \%$ of visual disturbances and 3.1\% from cardiovascular disorders (Figure 1).

Furthermore, $22.2 \%$ of respondents reveal cases of diabetes in their families including $10.1 \%$ among relatives.

The majority of respondents (70.5\%) have a healthy weight (BMI situated between 18.5 and $25 \mathrm{~kg} / \mathrm{m}^{2}$ ). Underweight concerned $14 \%$ of respondents. Their proportion is almost identical among men and women (respectively $14.3 \%$ and $13.6 \%$ ). The cases of extreme thinness are very rare (1.1\% in men and $2.6 \%$ for women). On the other side, $10.6 \%$ are overweight and $3.2 \%$ are obese. Overweight and obesity are more common among women: $16.8 \%$ and $7.2 \%$ against respectively $7.2 \%$ and $1.0 \%$ for men. Cases of morbid obesity (BMI > 40) were not observed (Figure 2).

Before our study, the majority of respondents (69\%) did not know their BMI and some had a wrong image of their body weight. Indeed, $40.9 \%$ of respondents with normal BMI considered themselves underweight or conversely, overweight. From 30.5\% who felt underweight, only half (14\%) really was (Figure 3 ).

Moreover, $36.4 \%$ of respondents were not satisfied with their body image. Among them, $68.3 \%$ would have liked to gain weight.

From a nutritional point of view, more than half of respondents (56.3\%) reported taking three meals a day, 39.8\% 2 meals and 3.9\% one meal. The vast majority $(86.3 \%)$ ate meals cooked within the family, against $13.7 \%$ who recurred to fast food. Food rations are composed of the major food groups, however, their frequencies in meals is very variable. For almost all students surveyed, cereals, mainly rice and starches, are the basis of the diet. Three-quarters (75.4\%) consume animal protein every day (mostly fish), 19.8\% consume them only 3 times a week and $4.5 \%$ only once a week. Dairy consumption is also very uneven: daily for $30.3 \%$ of respondents, tri-weekly for $30.4 \%$ and weekly for $24 \%$. The lipids are consumed daily by $87.3 \%$ of students essentially in the form of vegetable oils. Fruits and vegetables are

Table 1. Sample by age and sex categories (numbers and \%).

\begin{tabular}{|c|c|c|c|c|c|c|c|}
\hline & 20 and less & $21-22$ & $23-24$ & $25-26$ & $27-28$ & 29 and more & Total \\
\hline \multirow[t]{2}{*}{ WOMAN } & 141 & 99 & 68 & 31 & 7 & $\mathbf{0}$ & 346 \\
\hline & $40,8 \%$ & $28,6 \%$ & $19,7 \%$ & $9,0 \%$ & $2,0 \%$ & $0,0 \%$ & $100,0 \%$ \\
\hline \multirow[t]{2}{*}{ MEN } & 111 & 167 & 156 & 117 & 48 & 25 & 624 \\
\hline & $17,8 \%$ & $26,8 \%$ & $25,0 \%$ & $18,8 \%$ & $7,7 \%$ & $4,0 \%$ & $100,0 \%$ \\
\hline \multirow[t]{2}{*}{ TOTAL } & 252 & 266 & 224 & 148 & 55 & 25 & 970 \\
\hline & $26,0 \%$ & $27,4 \%$ & $23,1 \%$ & $15,3 \%$ & $5,7 \%$ & $2,6 \%$ & $100,0 \%$ \\
\hline
\end{tabular}




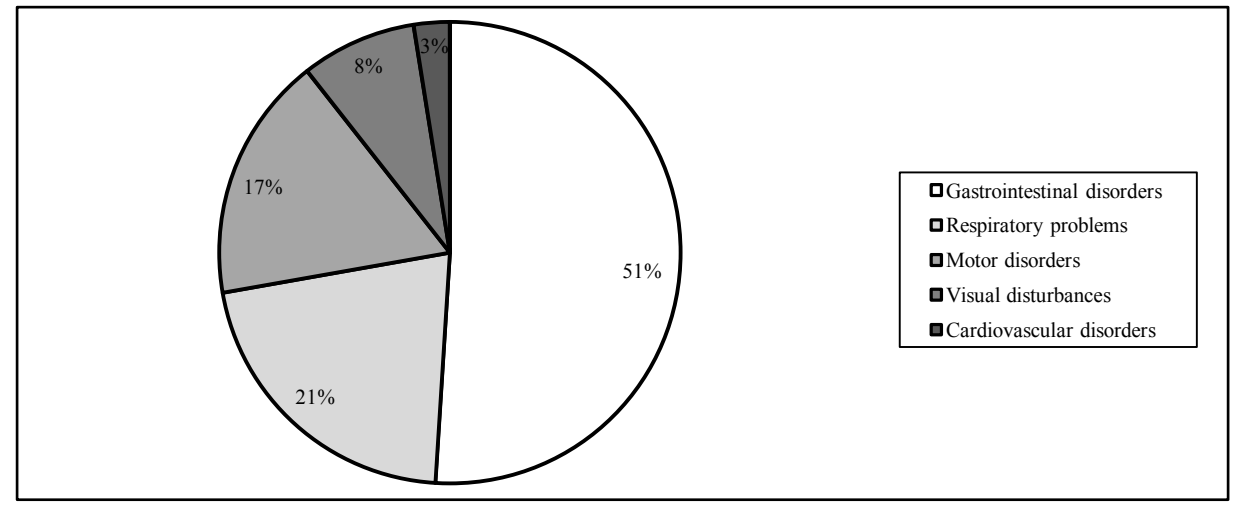

Figure 1. Frequency and nature of Medical problems.

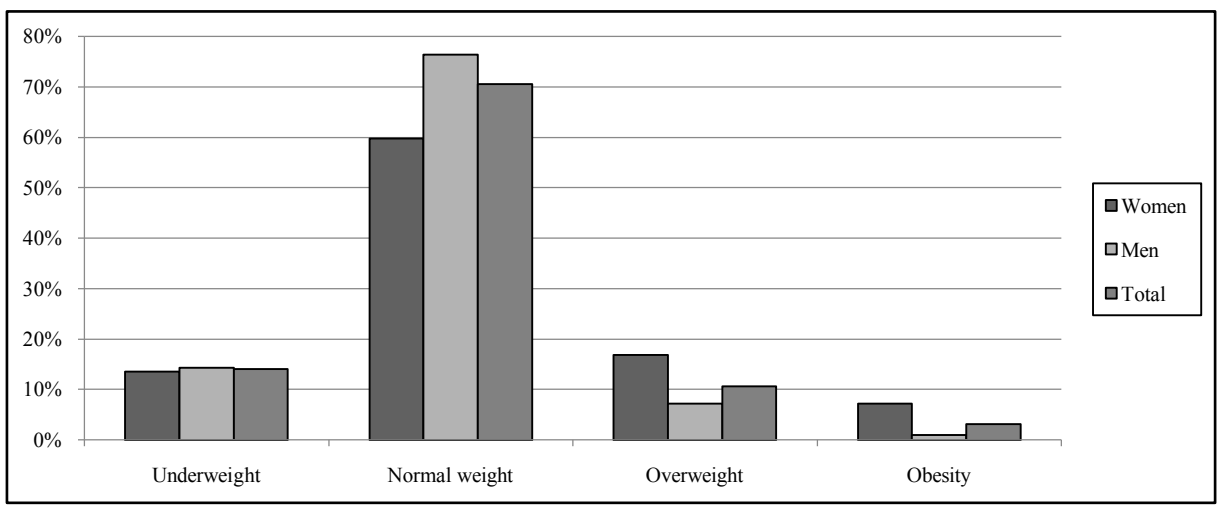

Figure 2. BMI by sexe.

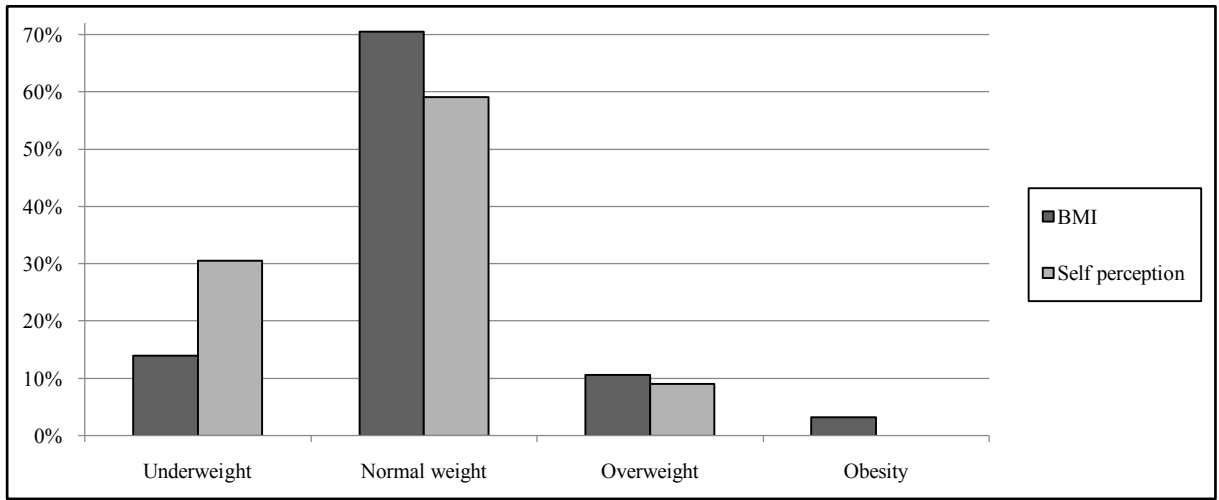

Figure 3. Comparison between BMI and self-perception of the body weight.

present in the daily ration for $35.7 \%$ of respondents. $35.4 \%$ consume them every two days, $24.3 \%$ once a week and $4.5 \%$ even more rarely (Figure 4).

Nevertheless, $85 \%$ of the surveyed students considered their nutrition as "proper or satisfying".

Almost none of the surveyed students had an employment and energy was only needed for motions related to everyday life and in some cases for recreational sport. Thus, over $80 \%$ of them rate their distance walked daily to less than $5 \mathrm{~km}$, which is less than an hour of walking per day. In addition, nearly $60 \%$ of their daily displacing was less than 3 kilometers. The women reported having a significantly lower perimeter walk than that of men (Figure 5).

Only $46.3 \%$ compensate for the lack of physical activity by one or more sport activities in the following frequency: 1 time per day (15.4\%), 3 times per week (37.9\%), once per week (43.3\%) and once per month (3.3\%). Regarding sports, football dominates all practices with $59.6 \%$. It is essentially informal matches that are played in the neighborhoods on improvised land without any supervision. $18.5 \%$ of the respondents practice running. Fitness and team sports (basketball and volleyball) were practiced by $10.3 \%$ and $7.1 \%$. Other sports are very rare and practice rate is insignificant. For non-practitioners, who constitute the majority of the sample (53.7\%), the forbearance reasons are numerous and varied: lack of time (67.5\%), uselessness $(25 \%)$ or the medical problems (7\%). Especially men evoked the lack of time; women considered sports more often "useless" than men. Medical reasons were mentioned with the same frequency in both sexes. The proportion of non-athletes largely predominated among women (83.5\% against $37.2 \%$ for men). 


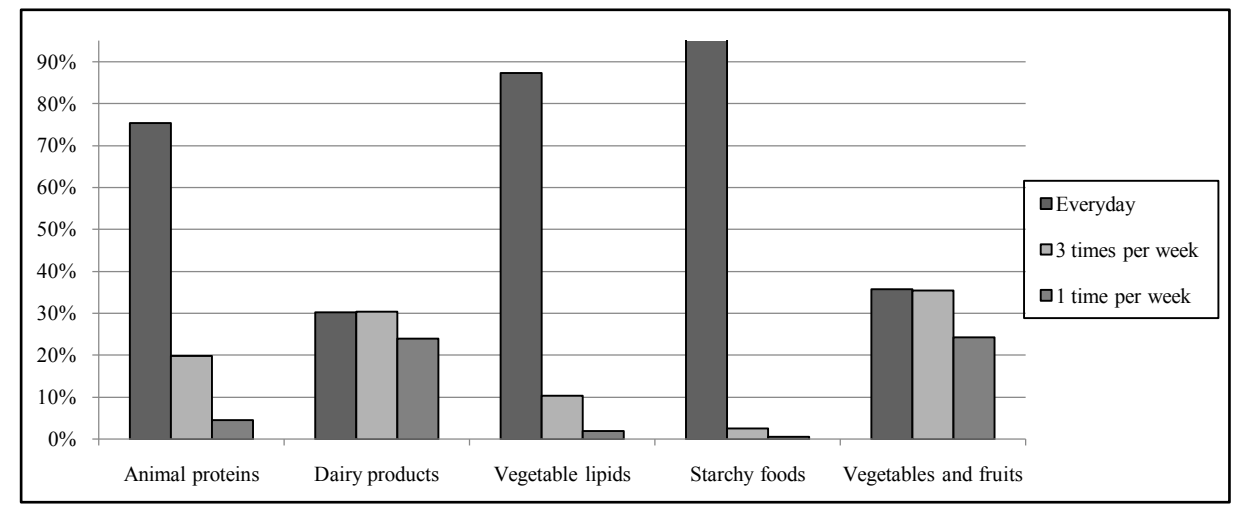

Figure 4. Frequency of consumption and composition of food rations.

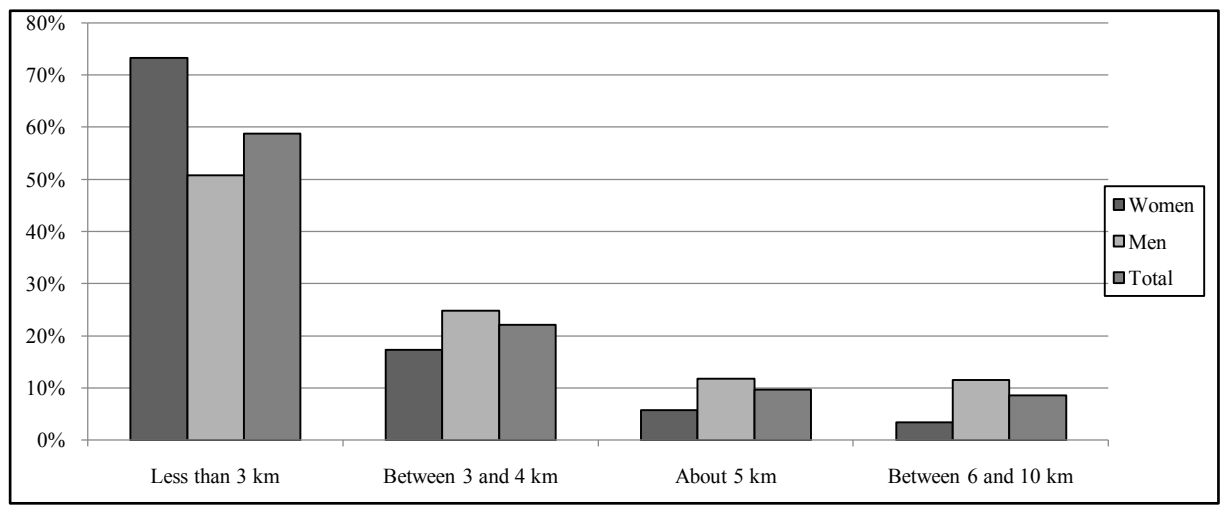

Figure 5. Walkingperimeter by sexe.

Finally, only $3.9 \%$ of the surveyed students (38 of 970 ) showed any of T2DM risk factors considered in this study. Just under a third (31.3\%) had one risk factor, $44.6 \%$ two of them, $17.5 \%$ and $2.3 \%$ had simultaneously three and four risk factors.

\section{Discussion and conclusion}

In this study, we collected data on diabetes and its risk factors among young adults residing in the Guinean capital Conakry. Because of its size ( $(\mathrm{=}=970)$ and diversity (age, sex, study level, place of birth ...), this sample is particularly representative of all medical students and more generally of students of the University of Conakry.

The analysis shows that the main risk factors of T2DM are malnutrition and lack of physical activity. In fact, the consumption of dairy products, fruits and vegetables is well below international recommendations. For example, the French National Nutrition and Health Program recommends daily consumption of at least 5 different fruits and vegetables, and 3 dairy products. Below this ration, the risk of development of T2DM increases significantly [20]. Almost all of the respondents however consume dairy products, vegetables and fruits only once to three times a week. As shown above, rice and starches are the basis of their diet. This scenario reflects an imbalance between "fast" sugars and fiber that may lead to T2DM.

With a sport rate of less than 3 times per week, or walked distances of less than $5 \mathrm{~km}$ per day, the level of energy expenditure of $63.6 \%$ of respondents appears to be under the physiological norms. According to the different epidemiological studies, in order to stay healthy, the minimum physical activity should be around thirty minutes of brisk walking per day or a minimum effort of 10000 daily steps. Below this threshold, the subject is considered to be at risk of developing noncommunicable diseases including T2DM (WHO, 2004).

The history of diabetes and overweight as a risk factor of T2DM represents respectively $22.2 \%$ and $13.2 \%$. If family history is a nonmodular risk factor that is independent of lifestyle, overweight can be attributed to physical inactivity and malnutrition. However, the representations of ideal body according to the surveyed subjects tend towards more corpulent bodies. It probably results from an under evaluation of the real weight compared to BMI. Indeed, traditions and local culture consider overweight to be a sign of health and wealth [21]. This scenario somehow explains the reluctance among some overweight subjects to lose weight. This socio-cultural context can be a real obstacle in the prophylaxis and treatment of T2DM and may contribute to its expansion.

In conclusion of this study, we can say that the context of globalization and social innovation forever changed the balance and lifestyles of young urban residents in Guinea. If the nutrition transition tends to gradually replace the traditional schemes with a less balanced diet that is richer in sugars and fats, cultural representations tend to feed the stereotypes of an ideal overweight body and to consider sports as useless. However, the problem does not only originate from changes in lifestyle, but also from the lack of proper canteens and sport facilities accessible and open to all. Without an investment in the future, Guinea, like most countries in the sub-region, now represents a "time bomb", where T2DM surely threatens to steadily increase.

\section{Funding}

This study was supported by the Faculty of Medicine of the University of Conakry. 


\section{References}

1. World Health Organization (WHO) (2012) Global status report on noncommunicable diseases 2014. Geneva: WHO.

2. Mathers CD, Loncar D (2006) Projections of global mortality and burden of disease from 2002 to 2030. PLoS Med 3: e442.[Crossref]

3. Zabsonré P, Sedogo B, Lankoande D, Dyemkouma FX, Bertrand Ed (2000) Obesity and chronicdiseases in Sub-Saharan Africa. Med Afr Noire 47: 5-9.

4. Gning SB, Thiam M, Fall F, et al. (2007) Diabetesmellitus in Sub-Saharan Africa. Epidemiological, supervisional difficulties. Médecine Tropicale 67: 607-611.

5. Baldé NM, Diallo I, Baldé MD, Barry IS, Kaba L, et al. (2007) Diabetes and impairedfasting glucose in rural and urban populations in FutaJallon (Guinea): prevalence and associated risk factors. Diabetes Metab 33: 114-20. [Crossref]

6. Bonita R, de Courten M, Dwyer T, Jamrozik K, Winkelmann R (2001) Surveillance of risk factors for noncommunicable disease: The WHO STEPwiseapproach. Geneva: World Health Organization.

7. Fagot-Campagna A, Narayan KM, Imperatore G (2001) Type 2 diabetes in children. BMJ 322: 377-378.[Crossref]

8. Alhyas L, McKay A, Balasanthiran A, Majeed A (2011) Prevalences of overweight, obesity, hyperglycaemia, hypertension and dyslipidaemia in the Gulf: systematicreview. JRSM Short Rep 2: 55.[Crossref]

9. Esayas HH, Hiroshi Y, Leo K, Atsuko A (2013) The genderdifferences in the prevalence of diabetesmellitus, abnormalfastingblood glucose and glucose intolerance in subSaharanAfrica: a systematicreview and meta-analysis. Bulletin of the World Health Organization. Geneva: WHO 91: 621-715.

10. Fagot-Campagna A, Romon I, Fosse S, Roudier C (2010) Prevalence and incidence of diabetes and diabetes-relatedmortality in France - EpidemiologicalSynthesis. French Institute for Public Health surveillance.
11. HealthAnalysis Division of Statistics Canada (2014) Diabetes. Avalable at www. statcan.gc.ca/pub/82-229-x/2009001/status/dia-fra.htm (accessed 12 Septembre 2015).

12. Sladek R, Rocheleau G, Rung J, Dina C, Shen L, et al. (2007) A genome-wide association study identifies novel risk loci for type 2 diabetes. Nature 445: 881-885. [Crossref]

13. King H, Aubert RE, Herman WH (1998) Global burden of diabetes, 1995-2025 prevalence, numericalestimates, and projections. Diabetes Care 21: 1414-1431. [Crossref]

14. Simon D, Bourgeon M, Balkau B, Eschwege E, Charles MA (2001) [Insulinsensitivity and ethnic groups]. Diabetes Metab 27: 215-221.[Crossref]

15. World Health Organization (WHO) (2009) Global healthrisks: mortality and burden of diseases attributable to selected major risks. Geneva: WHO. Available at http:/ www.who.int/healthinfo/global_burden_disease/GlobalHealthRisks_report_full.pdf (accessed 20 September 2015).

16. World Health Organization (WHO) (2004) Global Strategy on Diet, PhysicalActivity and Health. Geneva: WHO. Available at: http://www.who.int/dietphysicalactivity/pa/ en/ (accessed 12 September 2015)

17. Beran D, Besançon S, Bowis J (2006) Diabetes, a major public healthproblem for Africa. ReMed 33: 6-8.

18. Millar WJ, Young TK (2003) Evolution of diabetes: Prevalence, incidence and risk factors, Health Reports, product n ${ }^{\circ}$ 82-003.Statistics Canada Catalogue 14: 39-52.

19. Lebart L, Morineau A, Piron M (1995). Multidimensionalexploratorystatistics. Paris: Dunod.

20. Ministry of Health (2012) French National Nutrition and Health Program 2011-2015. Available at: http://www.sante.gouv.fr/IMG/pdf/PNNS UK INDD V2.pdf. (accessed 20 September 2015)

21. Correia J, Pataky Z, Golay A (2014) [Understandingobesity in Africa: the effect of [economic] development and [mental] concepts]. Rev Med Suisse 10: 712-716. [Crossref]

Copyright: C2017 CHIRKOV VT. This is an open-access article distributed under the terms of the Creative Commons Attribution License, which permits unrestricted use, distribution, and reproduction in any medium, provided the original author and source are credited. 